\title{
Vibrational Analysis of Peptides, Polypeptides, and Proteins. XXXII. $\alpha$-Poly(L-Glutamic Acid)
}

\author{
PRADEEP K. SENGUPTA and S. KRIMM, Biophysics Research \\ Division, University of Michigan, Ann Arbor, Michigan 48109
}

\begin{abstract}
Synopsis
The Raman and ir spectra of $\alpha$-helical poly(L-glutamic acid) have been assigned on the basis of a normal mode calculation for this structure. The force field was based on our previously refined main-chain force constants for $\alpha$-poly(L-alanine) and side-chain force constants for $\beta$-calcium-poly(L-glutamate). Despite the identical backbone $\alpha$-helical structures, significantly different frequencies are calculated, and observed, in the amide III and backbone stretch regions of a-poly(L-glutamic acid), as compared with $\alpha$ poly(L-alanine). This clearly demonstrates the influence of side-chain structure on mainchain vibrational modes.
\end{abstract}

\section{INTRODUCTION}

In a previous paper, ${ }^{1}$ we presented an analysis of the Raman and ir spectra of calcium poly(L-glutamate) $\left[(\mathrm{GluCa})_{n}\right]$ in the $\beta$-sheet conformation, based on normal mode calculations of the antiparallel-chain pleated-sheet structure. In this paper, we analyze the vibrational spectra of the $\alpha$-helix conformation of this polypeptide, particularly in the acid form, viz., poly(L-glutamic acid) $\left[(\mathrm{GluH})_{n}\right]$, again with the aid of normal mode calculations. Such calculations provide the most powerful method of correlating the vibrational spectrum with the conformation of a polypeptide chain. ${ }^{2}$ The results of this analysis not only help to interpret the Raman and ir spectra of $\alpha-(\mathrm{GluH})_{n}$, but they show how the chemical composition of the side chain influences the vibrations of the main chain. They also provide a basis for analyzing the conformation of the charged form of $(\mathrm{GluH})_{n}$ (P. K. Sengupta and S. Krimm, to be published).

The $\alpha-(\mathrm{GluH})_{n}$ conformation has been studied by ir and Raman techniques, both in the solid state ${ }^{3-8}$ and in solution..$^{9,10}$ The existence of a folded structure was first proposed by Ambrose $^{3}$ on the basis of ir dichroism measurements on solid films cast from solutions in dimethylformamide (DMF). One of the motivations for that study was to compare the structure of the synthetic polypeptide with that of the poly(D-glutamic acid) isolated from Bacillus anthracis. It was found that, while the synthetic material was exclusively in the $\alpha$-helix conformation, the natural polypeptide consisted of a mixture of $\alpha$-hellix and $\beta$-sheet conformations. Subsequent studies of the vibrational spec- 
tra of $(\mathrm{GluH})_{n}$ were related to the helix-coil transition of this polypeptide and its relevance to the processes of protein denaturation. These studies confirmed that $(\mathrm{GluH})_{n}$ adopts an $\alpha$-helix conformation in the solid state and in aqueous solutions at low $\mathrm{pH}$, i.e., under conditions where the $\mathrm{COOH}$ groups are nonionized. Recent Raman studies on $(\mathrm{GluCa})_{n}$ in the hexagonal crystalline form ${ }^{11}$ have indicated that both of these salts also adopt $\alpha$-helix conformations in the dry state.

While previous studies have focussed on the interpretation of isolated features of the ir and Raman spectra, we present here a detailed analysis based on a normal mode calculation of the $\alpha$-helix structure.

\section{MATERIALS AND METHODS}

The $(\mathrm{GluH})_{n}$ sample used in this study was obtained from Miles Laboratories, Inc. (Lot No. 802, $M=63,800$ ). For the ir measurements, films were cast from a DMF solution on to $\mathrm{AgBr}$ plates. A partially $\mathrm{N}$-deuterated sample was prepared as follows: $\mathrm{D}_{2} \mathrm{O}$ was added to a solution of $(\mathrm{GluH})_{n}$ in DMF. This was allowed to sit for several hours, after which the solvent was evaporated in a vacuum oven at room temperature. This sample was redissolved in DMF and the above procedure repeated two more times.

Most of the ir measurements were made at $2 \mathrm{~cm}^{-1}$ resolution on a Digilab FTS-20C Fourier transform ir spectrometer, with some measurements made on a Nicolet 60SX instrument. The common frequencies are in reasonably good agreement with literature values; we also present additional data in the $\mathrm{NH}$ and $\mathrm{CH}$ stretch regions, and weak bands in the $1800-400-\mathrm{cm}^{-1}$ region not listed by previous workers. The Raman data used in this study were taken from Fasman, et al., ${ }^{8}$ with the exception of the $\mathrm{CH}_{2}$ stretch region, which was measured in this laboratory using a Raman spectrometer described previously. ${ }^{1}$

\section{NORMAL MODE CALCULATIONS}

The normal mode calculation was done on an $\alpha$-helix conformation identical to that of $\alpha$-(Ala $)_{n}$, ${ }^{12}$ i.e., with helix parameters of $n=3.62$, $h=1.495 \AA$, and $t$ (rotation per residue) $=99.57^{\circ}$, leading to dihedral angles of $\phi=-57.37^{\circ}$ and $\psi=-47.49^{\circ}$. The side chains were assumed to be fully extended, and their dihedral angles were taken from the comparable structure in $\beta$-(GluCa $)_{n}{ }^{1}$ For convenience, the $\mathrm{COOH}$ group was replaced by a $\mathrm{COO}^{-}$group; this should have a small effect on the main-chain modes, but may influence some side-chain frequencies. (We will identify specific COOH bands in the spectra.) The internal and local symmetry coordinates were defined as before, ${ }^{1}$ except that in the present case we do not require the $\mathrm{H}^{\alpha} \cdots \mathrm{H}^{\alpha}$ stretching coordinate. 
The vibrationally active modes of the $\alpha$-helix are classified into $\mathrm{A}(\delta$ $\left.=0^{\circ}\right), \mathrm{E}_{1}\left(\delta=99.57^{\circ}\right)$, and $\mathrm{E}_{2}\left(\delta=199.04^{\circ}\right)$ symmetry species, where $\delta$ is the phase difference between the motions in adjacent residues. The $A$ and $E_{1}$ species modes are both ir and Raman active and have parallel and perpendicular ir dichroism, respectively. The $E_{2}$ species modes are Raman active only. For our structure, there are $43 \mathrm{~A}, 44 \mathrm{E}_{1}$, and 45 $\mathrm{E}_{2}$ modes.

The force constants for the main chain and those involving the coupling of the $\mathrm{C}^{\beta}$ atom to the backbone were taken from the work on $\alpha$-(Ala) ${ }_{n}{ }^{12}$ The force constants for the side chain were transferred from $\beta$-(GluCa $)_{n}{ }^{1}$ As in the previous calculation for the $\alpha$-helix, ${ }^{12}$ transition dipole coupling was included for amide I and amide II modes, leading to calculated splittings identical to those for $\alpha$-(Ala $)_{n}$.

\section{RESULTS AND DISCUSSION}

The ir spectra of a solid film of $\alpha-(\mathrm{GluH})_{n}$ are given in Figs. 1 and $2 \mathrm{~A}$, with the spectrum of a partially $\mathrm{N}$-deuterated sample being given in Fig. 2B. The observed Raman ${ }^{8}$ and ir bands, together with the calculated frequencies and their potential energy distributions (PED), are given in Table I.

The amide A mode, which is NH stretch perturbed by Fermi resonance, is observed at $3301 \mathrm{~cm}^{-1}$, close to the value of $3307 \mathrm{~cm}^{-1}$ found in $\alpha$-(Ala) ${ }_{n}{ }^{13}$ (We must point out that the $\mathrm{OH}$ stretch mode of the

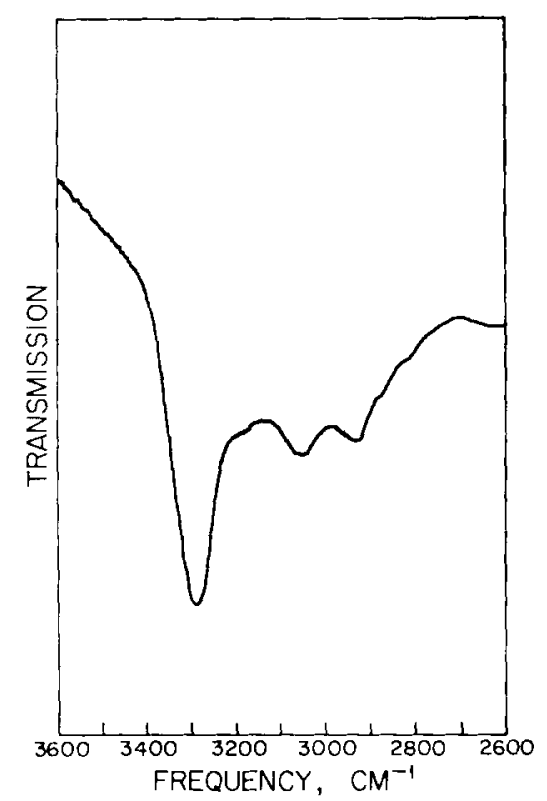

Fig. 1. The ir spectrum of $a$-poly(L-glutamic acid) in the $2600-3600-\mathrm{cm}^{-1}$ region. 


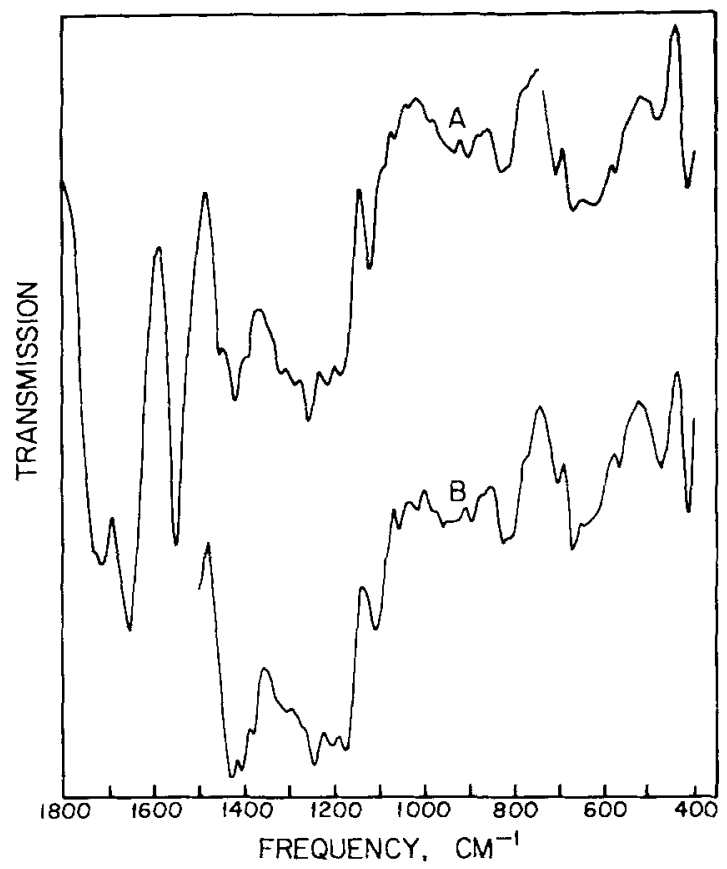

Fig. 2. The ir spectrum of $\alpha$-poly(L-glutamic acid) in the $400-1800-\mathrm{cm}^{-1}$ region: (A) parent compound, (B) partially $\mathrm{N}$-deuterated derivative.

$\mathrm{COOH}$ group is expected in the same general region as $\mathrm{NH}$ stretch, but no conspicuous sharp band is observed that can be readily assigned to this mode.) The amide $B$ mode, which is the other component of the Fermi resonance doublet, is observed at $3067 \mathrm{~cm}^{-1}$, compared with $3058 \mathrm{~cm}^{-1}$ in $\alpha-(\mathrm{Ala})_{n} \cdot{ }^{13}$ If the resonance involves the fundamental and the overtone of the $\mathrm{E}_{1}$ species amide II mode, ${ }^{13}$ then the fundamental is expected at $3301-(2 \times 1550-3067)=3268 \mathrm{~cm}^{-1}$. Even allowing

TABLE I

Observed and Calculated Frequencies (in $\mathrm{cm}^{-1}$ ) of $\alpha$-Poly(L-Glutamic Acid)

\begin{tabular}{|c|c|c|c|c|c|}
\hline \multicolumn{2}{|c|}{ Observed $^{a}$} & \multicolumn{4}{|r|}{ Calculated } \\
\hline Raman $^{\mathrm{b}}$ & ir & A & $\mathrm{E}_{1}$ & $\mathrm{E}_{2}$ & Potential Energy Distribution $^{\mathrm{c}}$ \\
\hline \multirow{10}{*}{$\sim 2933 \mathrm{VS}$} & $3301 \mathrm{VS}^{d}$ & 3279 & & & NH s(98) \\
\hline & & & 3279 & & $\mathrm{NH} \mathbf{s}(98)$ \\
\hline & & & & 3279 & NH s(98) \\
\hline & & 2982 & & & $\mathrm{C}^{\gamma} \mathrm{H}_{2}$ as $(99)$ \\
\hline & \multirow{6}{*}{$2939 \mathrm{M}$} & & 2981 & & $\mathrm{C}^{\gamma} \mathrm{H}_{2}$ as(99) \\
\hline & & & & 2981 & $\mathrm{C}^{\gamma} \mathrm{H}_{2}$ as $(99)$ \\
\hline & & 2943 & & & $\mathrm{C}^{\gamma} \mathrm{H}_{2} \mathrm{ss}(99)$ \\
\hline & & & 2943 & & $\mathrm{C}^{\gamma} \mathrm{H}_{2} \mathrm{ss}(99)$ \\
\hline & & & & 2943 & $\mathrm{C}^{\gamma} \mathrm{H}_{2} \mathbf{s s}(99)$ \\
\hline & & 2918 & & & $\mathrm{C}^{\beta} \mathrm{H}_{2}$ as $(98)$ \\
\hline
\end{tabular}


TABLE I (Continued)

Observed and Calculated Frequencies (in $\mathrm{cm}^{-1}$ ) of $\alpha$-Poly(L-Glutamic Acid)

\begin{tabular}{|c|c|c|c|c|c|}
\hline \multicolumn{2}{|c|}{ Observed ${ }^{\mathrm{a}}$} & \multicolumn{4}{|r|}{ Calculated } \\
\hline $\operatorname{Raman}^{\mathrm{b}}$ & ir & A & $E_{1}$ & $\mathbf{E}_{2}$ & Potential Energy Distribution ${ }^{c}$ \\
\hline \multirow{7}{*}{$\sim 2883 W$} & \multirow{7}{*}{$\sim 2880 \mathrm{sh}$} & & 2918 & & $\mathrm{C}^{\beta} \mathrm{H}_{2}$ as $(98)$ \\
\hline & & & & 2918 & $\mathrm{C}^{\beta} \mathrm{H}_{2}$ as $(98)$ \\
\hline & & 2884 & & & $\mathrm{C}^{\alpha} \mathrm{H}^{\alpha} \mathbf{s}(98)$ \\
\hline & & & 2884 & & $\mathrm{C}^{\alpha} \mathrm{H}^{\alpha} \mathrm{s}(98)$ \\
\hline & & & & 2884 & $\mathrm{C}^{\alpha} \mathrm{H}^{\alpha} \mathrm{s}(98)$ \\
\hline & & 2855 & & & $\mathrm{C}^{\beta} \mathrm{H}_{2} \mathrm{ss}(98)$ \\
\hline & & & 2855 & & $\mathrm{C}^{\beta} \mathrm{H}_{2} \mathrm{ss}(98)$ \\
\hline \multirow[t]{2}{*}{$1720^{e}$} & $1718 \mathrm{~S}^{\mathrm{e}}$ & & & 2855 & $\mathrm{C}^{\beta} \mathrm{H}_{2} \mathrm{ss}(98)$ \\
\hline & $1653 V_{S}^{f}$ & 1657 & & & $\begin{array}{l}v\left(\mathrm{C}^{\circ}=0\right)\left[1739\left(\mathrm{~B}_{\mathrm{u}}\right)\right] \\
\mathrm{CO} \mathrm{s}(82), \mathrm{CN} \mathrm{s}(10), \mathrm{C}^{\mathrm{a}} \mathrm{CN} \mathrm{d}(10)\end{array}$ \\
\hline \multirow[t]{9}{*}{$1652 S$} & & & 1655 & & $\mathrm{CO} s(82), \mathrm{CN} s(11), \mathrm{C}^{\alpha} \mathrm{CN} d(10)$ \\
\hline & & & & 1645 & $\mathrm{CO} \mathrm{s}(83), \mathrm{CN} s(12), \mathrm{C}^{a} \mathrm{CN} \mathrm{d}(10)$ \\
\hline & & 1561 & & & $\mathrm{C}^{6} \mathrm{O}_{2}$ as $(103)$ \\
\hline & & & 1561 & & $\mathrm{C}^{8} \mathrm{O}_{2}$ as $(103)$ \\
\hline & & & & 1561 & $\mathrm{CaO}_{2}$ as $(103)$ \\
\hline & & & & 1539 & $\begin{array}{l}\text { NH ib }(46), \mathrm{CN} s(31), \mathrm{CO} \mathrm{ib}(12), \\
\quad \mathrm{C}^{\alpha} \mathrm{C} s(11)\end{array}$ \\
\hline & $1550 S_{\downarrow}$ & & 1537 & & $\begin{array}{l}\text { NH ib }(46), \mathrm{CN} s(33), \mathrm{CO} \mathrm{ib}(11), \\
\quad \mathrm{C}{ }^{\circ} \mathrm{C} \mathrm{s}(10)\end{array}$ \\
\hline & $1510 W_{\|}^{g}$ & 1517 & & & NH ib(46),CN s(35),CO ib(11) \\
\hline & & & & 1459 & $\mathrm{C}^{\beta} \mathrm{H}_{2} \mathrm{~b}(52), \mathrm{C}^{\gamma} \mathrm{H}_{2} \mathrm{~b}(44)$ \\
\hline \multirow[t]{2}{*}{$1450 \mathrm{M}$} & $1451 W_{\perp}$ & & 1459 & & $\mathrm{C}^{\beta} \mathrm{H}_{2} \mathrm{~b}(51), \mathrm{C}^{\gamma} \mathrm{H}_{2} \mathrm{~b}(45)$ \\
\hline & & 1458 & & & $\mathrm{C}^{\beta} \mathrm{H}_{2} \mathrm{~b}(50), \mathrm{C}^{\gamma} \mathrm{H}_{2} \mathrm{~b}(47)$ \\
\hline \multirow[t]{12}{*}{$1420 \mathrm{M}$} & $1417 \mathrm{M}$ & 1445 & & & $\mathrm{C}^{\gamma} \mathrm{H}_{2} \mathrm{~b}(48), \mathrm{C}^{\beta} \mathrm{H}_{2} \mathrm{~b}(41)$ \\
\hline & 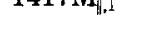 & & 1445 & & $\mathrm{C}^{\gamma} \mathrm{H}_{2} \mathrm{~b}(49), \mathrm{C}^{\beta} \mathrm{H}_{2} \mathrm{~b}(39)$ \\
\hline & & & & 1445 & $\mathrm{C}^{\gamma} \mathrm{H}_{2} \mathrm{~b}(51), \mathrm{C}^{\beta} \mathrm{H}_{2} \mathrm{~b}(37)$ \\
\hline & & 1416 & & & $\mathrm{C}^{8} \mathrm{O}_{2} \mathrm{ss}(67), \mathrm{C}^{a} \mathrm{O}_{2} \mathrm{~b}(24), \mathrm{C}^{\gamma} \mathrm{C}^{6} \mathrm{~s}(15)$ \\
\hline & & & 1416 & & $\mathrm{C}^{8} \mathrm{O}_{2} \mathrm{ss}(67), \mathrm{C}^{8} \mathrm{O}_{2} \mathrm{~b}(24), \mathrm{C}^{\gamma} \mathrm{C}^{\delta} \mathrm{s}(15)$ \\
\hline & & & & 1416 & $\mathrm{C}^{8} \mathrm{O}_{2} \mathrm{ss}(67), \mathrm{C}^{\delta} \mathrm{O}_{2} \mathrm{~b}(24), \mathrm{C}^{\gamma} \mathrm{C}^{\delta} \mathrm{s}(15)$ \\
\hline & & & & 1383 & $\begin{array}{l}\mathrm{C}^{\beta} \mathrm{H}_{2} \mathrm{w}(35), \mathrm{H}^{\alpha} \text { b1 }(25), \mathrm{C}^{\alpha} \mathrm{C}^{\beta} \mathrm{s}(13) \\
\quad \mathrm{C}^{\beta} \mathrm{C}^{\gamma} \mathrm{s}(12)\end{array}$ \\
\hline & $1388 \mathrm{~W}$ & & 1380 & & $\mathrm{C}^{\beta} \mathrm{H}_{2} \mathrm{w}(36), \mathrm{H}^{\alpha}$ b1 $(22), \mathrm{C}^{\beta} \mathrm{C}^{\gamma} \mathrm{s}(13)$ \\
\hline & & 1375 & & & $\begin{array}{l}\mathrm{C}^{\beta} \mathrm{H}_{2} \mathrm{w}(41), \mathrm{H}^{\alpha} \text { b1 }(18), \mathrm{C}^{A} \mathrm{C}^{\gamma} \mathrm{s}(15) \\
\quad \mathrm{C}^{a} \mathrm{C}^{\beta} \mathrm{s}(12)\end{array}$ \\
\hline & & 1343 & & & $\mathrm{C}^{\beta} \mathrm{H}_{2} \operatorname{tw}(32), \mathrm{H}^{\alpha} \mathrm{b} 2(29)$ \\
\hline & & & 1341 & & $\mathrm{C}^{\beta} \mathrm{H}_{2} \mathrm{tw}(33), \mathrm{H}^{a} \mathrm{~b} 2(20), \mathrm{C}^{\gamma} \mathrm{H}_{2} \mathrm{tw}(10)$ \\
\hline & & & & 1339 & $\mathrm{C}^{\beta} \mathrm{H}_{2} \operatorname{tw}(41), \mathrm{C}^{\gamma} \mathrm{H}_{2} \operatorname{tw}(13), \mathrm{H}^{\alpha} \mathrm{b} 2(10)$ \\
\hline \multirow[t]{3}{*}{$1340 \mathrm{M}$} & $1343 \mathrm{sh}$ & & 1326 & & $\begin{array}{l}\mathrm{C}^{\gamma} \mathrm{H}_{2} \mathrm{w}(20), \mathrm{H}^{a} \text { b2(16), } \mathrm{NH} \mathrm{ib}(11) \\
\mathrm{C}^{\beta} \mathrm{H}_{2} \mathrm{tw}(10)\end{array}$ \\
\hline & & 1324 & & 1326 & $\begin{array}{l}\mathrm{C}^{\gamma} \mathrm{H}_{2} \mathrm{w}(23), \mathrm{NH} \mathrm{ib}(19), \mathrm{C}^{\alpha} \mathrm{C} \mathrm{s}(12) \\
\mathrm{H}^{\alpha} \mathrm{b} 2(26), \mathrm{C}^{\gamma} \mathrm{H}_{2} \mathrm{w}(18), \mathrm{C}^{\beta} \mathrm{H}_{2} \mathrm{tw}(11)\end{array}$ \\
\hline & & & & 1311 & $\mathrm{H}^{\alpha} \mathrm{b} 2(47), \mathrm{C}^{\gamma} \mathrm{H}_{2} \mathrm{w}(21)$ \\
\hline \multirow[t]{2}{*}{$\sim 1310 \mathrm{~W}, \mathrm{br}$} & $1313 W$ & & 1307 & & $\begin{array}{l}\mathrm{C}^{\gamma} \mathrm{H}_{2} \mathrm{w}(26), \mathrm{H}^{a} \mathrm{~b} 2(20), \mathrm{H}^{\mathrm{a}} \mathrm{b} 1(16) \\
\quad \mathrm{C}^{\gamma} \mathrm{H}_{2} \mathrm{tw}(13)\end{array}$ \\
\hline & & 1305 & & & $\mathrm{C}^{\gamma} \mathrm{H}_{2} \mathrm{w}(30), \mathrm{H}^{\alpha}$ b1 $(21), \mathrm{C}^{\gamma} \mathrm{H}_{2} \mathrm{tw}(20)$ \\
\hline \multirow[t]{3}{*}{$1296 \mathrm{M}$} & & & & 1299 & $\mathrm{H}^{a} \mathrm{~b} 2(24), \mathrm{C}^{\gamma} \mathrm{H}_{2} \mathrm{tw}(23), \mathrm{NH} \mathrm{ib}(15)$ \\
\hline & $1283 W_{\perp}$ & & 1287 & & $\mathrm{H}^{a} \mathrm{~b} 2(29), \mathrm{NH} \mathrm{ib}(25), \mathrm{C}^{\gamma} \mathrm{H}_{2} \mathrm{tw}(11)$ \\
\hline & & 1263 & & & $\begin{array}{l}\text { NH ib }(37), \mathrm{H}^{a} \text { b2(23),CN s(10), } \\
\quad \text { CO ib(10) }\end{array}$ \\
\hline
\end{tabular}


TABLE I (Continued)

Observed and Calculated Frequencies (in $\mathrm{cm}^{-1}$ ) of $\alpha$-Poly(L-Glutamic Acid)

\begin{tabular}{|c|c|c|c|c|c|}
\hline \multicolumn{2}{|c|}{ Observed $^{a}$} & \multicolumn{4}{|r|}{ Calculated } \\
\hline $\operatorname{Raman}^{\mathrm{b}}$ & ir & A & $\mathrm{E}_{1}$ & $\mathrm{E}_{2}$ & Potential Energy Distribution ${ }^{\mathfrak{c}}$ \\
\hline \multirow{5}{*}{$1214 \mathrm{~W}$} & $1253 \mathrm{MS}^{\mathrm{d}}$ & \multirow{4}{*}{1239} & & & $v(\mathrm{C}-\mathrm{O})\left[1255\left(\mathrm{~A}_{\mathrm{g}}\right)\right]$ \\
\hline & & & & & $\mathrm{O}^{\beta} \mathrm{H}_{2} \mathrm{w}(37), \mathrm{C}^{\gamma} \mathrm{H}_{2} \mathrm{w}(20), \mathrm{H}^{\alpha} \mathrm{b} 2(10)$ \\
\hline & $1212 \mathbf{W}_{\|, 1}$ & & & & \\
\hline & & & 1237 & & $\mathrm{C}^{\beta} \mathrm{H}_{2} \mathrm{w}(43), \mathrm{C}^{\gamma} \mathrm{H}_{2} \mathrm{w}(23)$ \\
\hline & & \multirow{3}{*}{1173} & & 1236 & $\begin{array}{l}\mathrm{H}^{\alpha} \text { 触(45), } \mathrm{C}^{\beta} \mathrm{H}_{2} \operatorname{tw}(22), \mathrm{C}^{\gamma} \mathrm{H}_{2} \operatorname{tw}(19)\end{array}$ \\
\hline \multirow[t]{2}{*}{$1178 \mathrm{~W}$} & $1181 W_{1}$ & & 1166 & & $\mathrm{H}^{\alpha} \mathrm{b} 1(29), \mathrm{C}^{\beta} \mathrm{H}_{2} \operatorname{tw}(28), \mathrm{C}^{\gamma} \mathrm{H}_{2} \operatorname{tw}(28)$ \\
\hline & & & & 1164 & $\mathrm{C}^{\gamma} \mathrm{H}_{2} \operatorname{tw}(33), \mathrm{C}^{\beta} \mathrm{H}_{2} \operatorname{tw}(31), \mathrm{H}^{\alpha}$ bl (25) \\
\hline \multirow[t]{2}{*}{$1117 \mathrm{~W}$} & $1118 \mathbf{M}_{\|, 1}$ & \multirow[t]{2}{*}{1129} & 1129 & & $\begin{array}{l}N^{\alpha} s(31), C^{\alpha} C^{\beta} s(20), C^{\gamma} H_{2} \operatorname{tw}(16) \\
C^{\alpha} C^{\beta} s(29), N^{\alpha} s(28)\end{array}$ \\
\hline & & & & 1127 & $\mathrm{C}^{\alpha} \mathrm{C}^{\beta} \mathrm{s}(35), \mathrm{NC}^{\alpha} \mathrm{s}(25), \mathrm{H}^{\alpha} \mathrm{b} 1(11)$ \\
\hline \multirow[t]{4}{*}{$1080 \mathrm{M}$} & $1083 W_{\sharp}$ & \multirow[t]{3}{*}{1085} & & & $\mathrm{C}^{\alpha} \mathrm{C}^{\beta} \mathrm{s}(46)$ \\
\hline & & & 1079 & & $\mathrm{C}^{\alpha} \mathrm{C}^{\beta} \mathrm{s}(30)$ \\
\hline & & & & 1075 & $\mathrm{C}^{\alpha} \mathrm{C}^{\beta} \mathrm{s}(21), \mathrm{C}^{\beta} \mathrm{C}^{\gamma} \mathrm{s}(11), \mathrm{C}^{\beta} \mathrm{H}_{2}(10)$ \\
\hline & & & & 1048 & $\mathrm{C}^{\beta} \mathrm{C}^{\gamma} \mathrm{s}(48)$ \\
\hline \multirow{2}{*}{$1057 \mathrm{M}$} & & \multirow{4}{*}{1047} & 1048 & & $\mathrm{C}^{\beta} \mathrm{C}^{\gamma} \mathbf{s}(50)$ \\
\hline & $1000 \mathbf{w}_{\|, 1}$ & & & & $\mathrm{C}^{\beta} \mathrm{C}^{\gamma} \mathrm{s}(51)$ \\
\hline $1030 \mathrm{~W}$ & $1028 \mathrm{~W}$ & & & & $?$ \\
\hline \multirow{24}{*}{$1002 \mathrm{~W}$} & & & & 998 & $\mathrm{C}^{\gamma} \mathrm{C}^{\delta} \mathrm{s}(26), \mathrm{C}^{\beta} \mathrm{C}^{\gamma} \mathrm{s}(10), \mathrm{C}^{\alpha} \mathrm{C}^{\beta} \mathrm{C}^{\gamma} \mathrm{d}(10)$ \\
\hline & $982 W$ & & 996 & & $\mathrm{C}^{\gamma} \mathrm{C}^{\delta} \mathrm{s}(28), \mathrm{C}^{\beta} \mathrm{C}^{\gamma} \mathrm{s}(10)$ \\
\hline & . & \multirow{5}{*}{$\begin{array}{l}992 \\
975\end{array}$} & & & $\mathrm{C}^{\gamma} \mathrm{C}^{\delta} \mathrm{s}(33), \mathrm{C}^{\beta} \mathrm{C}^{\gamma} \mathrm{s}(10), \mathrm{C}^{\delta} \mathrm{O}_{2} \mathrm{ss}(10)$ \\
\hline & & & & & $\begin{array}{l}\mathrm{C}^{\gamma} \mathrm{H}_{2} \mathrm{r}(28), \mathrm{NC}^{\alpha} \mathrm{s}(18), \mathrm{C}^{\beta} \mathrm{H}_{2} \mathrm{tw}(12), \\
\quad \mathrm{C}^{\beta} \mathrm{H}_{2} \mathrm{r}(11), \mathrm{C}^{\alpha} \mathrm{C} \mathbf{s}(10)\end{array}$ \\
\hline & & & 972 & & $\begin{array}{l}\mathrm{C}^{\gamma} \mathrm{H}_{2} \mathbf{r}(26), \mathrm{NC}^{\alpha} \mathrm{s}(14), \mathrm{C}^{\beta} \mathrm{H}_{2} \mathrm{tw}(11) \\
\quad \mathrm{C}^{\beta} \mathrm{H}_{2} \mathrm{r}(11)\end{array}$ \\
\hline & & & & 969 & $\begin{array}{l}\mathrm{C}^{\gamma} \mathrm{H}_{2} \mathrm{r}(26), \mathrm{NC}^{\alpha} \mathrm{s}(14), \mathrm{C}^{\beta} \mathrm{H}_{2} \mathrm{tw}(11), \\
\quad \mathrm{C}^{\beta} \mathrm{H}_{2} \mathrm{r}(10)\end{array}$ \\
\hline & & & & 934 & $\mathrm{CN} s(21), \mathrm{C}^{\alpha} \mathrm{C} s(17)$ \\
\hline & $928 \mathrm{~W}$ br & \multirow{3}{*}{922} & 929 & & $\mathrm{CN} s(23), \mathrm{C}^{a} \mathrm{C} \mathrm{s}(13)$ \\
\hline & & & & & $\mathrm{CN} s(27), \mathrm{CNC}^{a} \mathrm{~d}(12)$ \\
\hline & $\left.\begin{array}{l}896 W^{e} \\
869 W^{e}\end{array}\right\}$ & & & & $v\left(\mathrm{C}^{\gamma}-\mathrm{COOH}\right)\left[898\left(\mathrm{~A}_{\mathrm{g}}\right), 860\left(\mathrm{~B}_{\mathrm{u}}\right)\right]$ \\
\hline & $\sim 824 \mathrm{MW}$ & \multirow{3}{*}{810} & 812 & & $\mathrm{C}^{\beta} \mathrm{H}_{2} \mathbf{r}(34), \mathrm{C}^{\gamma} \mathrm{H}_{2} \mathbf{r}(24)$ \\
\hline & $\sim 810 \mathrm{MW}$ & & & & $\mathrm{C}^{\beta} \mathrm{H}_{2} \mathrm{r}(38), \mathrm{C}^{\gamma} \mathrm{H}_{2} \mathrm{r}(27)$ \\
\hline & \multirow{7}{*}{$768 \mathrm{VW}$} & & & 807 & $\mathrm{C}^{\beta} \mathrm{H}_{2} \mathrm{r}(46), \mathrm{C}^{\gamma} \mathrm{H}_{2} \mathrm{r}(30)$ \\
\hline & & 2 & 783 & & $\mathrm{CO} \mathrm{ob}(25), \mathrm{C}^{\beta} \mathrm{H}_{2} \mathrm{r}(19)$ \\
\hline & & \multirow[t]{2}{*}{777} & & & $\mathrm{C}^{\beta} \mathrm{H}_{2} \mathrm{r}(13), \mathrm{C}^{\delta} \mathrm{O}_{2} \mathrm{~b}(12), \mathrm{C}^{\beta} \mathrm{b} 1(10)$ \\
\hline & & & & 767 & $\mathrm{CO}$ ob(46) \\
\hline & & \multirow[t]{3}{*}{746} & & & $\mathrm{CO} \mathrm{ob}(35), \mathrm{CN} t(31)$ \\
\hline & & & 725 & & $\mathrm{C}^{8} \mathrm{O}_{2} \mathrm{~b}(38), \mathrm{C}^{8} \mathrm{O}_{2} \mathrm{ss}(12)$ \\
\hline & & & & 720 & $\mathrm{C}^{6} \mathrm{O}_{2} \mathrm{~b}(44), \mathrm{C}^{6} \mathrm{O}_{2} \mathrm{ss}(14)$ \\
\hline & \multirow[t]{2}{*}{$705 \mathrm{~W}$} & \multirow[t]{2}{*}{706} & & & $\begin{array}{l}\mathrm{C}^{\delta} \mathrm{O}_{2} \mathrm{~b}(24), \mathrm{NC}^{a} \mathrm{C} \mathrm{d}(15), \mathrm{C}^{\delta} \mathrm{O}_{2} \mathrm{w}(12) \\
\mathrm{C}^{\alpha} \mathrm{CN} \mathrm{d}(11), \mathrm{C}^{\beta} \mathrm{C}^{\gamma} \mathrm{C}^{\delta} \mathrm{d}(11)\end{array}$ \\
\hline & & & & 694 & $\mathrm{CO} \mathrm{ib}(18), \mathrm{CN} \mathrm{t}(14)$ \\
\hline & \multirow[t]{3}{*}{$\sim 670 \mathrm{MW}$} & & 678 & & $\mathrm{CN} t(19), \mathrm{NC}^{a} \mathrm{C} \mathrm{d}(12), \mathrm{NH}$ ob(11) \\
\hline & & & & 643 & 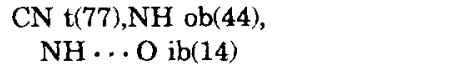 \\
\hline & & 628 & & & $\begin{array}{l}\mathrm{C}^{8} \mathrm{O}_{2} \mathrm{w}(26), \mathrm{C}^{8} \mathrm{O}_{2} \mathrm{~b}(20), \mathrm{CN} \mathrm{t}(20) \\
\mathrm{C}^{\gamma} \mathrm{C}^{\delta} \mathrm{s}(10)\end{array}$ \\
\hline
\end{tabular}


TABLE I (Continued)

Observed and Calculated Frequencies (in $\mathrm{cm}^{-1}$ ) of $\alpha$-Poly(L-Glutamic Acid)

\begin{tabular}{|c|c|c|c|c|c|}
\hline \multicolumn{2}{|c|}{ Observed $^{\mathrm{a}}$} & \multicolumn{4}{|r|}{ Calculated } \\
\hline Raman $^{b}$ & ir & A & $\mathbf{E}_{1}$ & $\mathbf{E}_{2}$ & Potential Energy Distribution ${ }^{c}$ \\
\hline & $618 \mathrm{MW}$ & & 626 & & $\begin{array}{l}\mathrm{CN} \mathrm{t}(42), \mathrm{C}^{\delta} \mathrm{O}_{2} \mathrm{w}(19), \mathrm{NH} \text { ob }(17) \\
\quad \mathrm{C}^{\delta} \mathrm{O}_{2} \mathrm{~b}(10)\end{array}$ \\
\hline & & & & 612 & $\mathrm{C}^{\delta} \mathrm{O}_{2} \mathrm{w}(46), \mathrm{C}^{\delta} \mathrm{O}_{2} \mathrm{~b}(13)$ \\
\hline & & & 608 & & $\begin{array}{l}\mathrm{C}^{\circ} \mathrm{O}_{2} \mathrm{w}(26), \mathrm{CN} \mathrm{t}(21), \mathrm{NH} \text { ob(14), } \\
\quad \mathrm{CO} \text { ob(12) }\end{array}$ \\
\hline & & 581 & & & $\begin{array}{l}\mathrm{C}^{8} \mathrm{O}_{2} \mathrm{w}(31), \mathrm{CN} \mathrm{t}(29), \mathrm{NH} \text { ob }(25) \\
\mathrm{CO} \mathrm{ob}(24)\end{array}$ \\
\hline \multirow[t]{5}{*}{$562 W$} & $567 \mathrm{~W}$ & 549 & & & 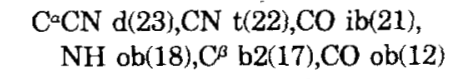 \\
\hline & & & 515 & & $\mathrm{NC}^{\alpha} \mathrm{C} \mathrm{d}(23), \mathrm{C}^{\alpha} \mathrm{CN} \mathrm{d}(13)$ \\
\hline & & & & 500 & $\mathrm{C}^{\circ} \mathrm{O}_{2} \mathrm{r}(89)$ \\
\hline & & 498 & & & $\mathrm{C}^{6} \mathrm{O}_{2} \mathrm{r}(88)$ \\
\hline & & & 498 & & $\mathrm{C}^{\circ} \mathrm{O}_{2} \mathrm{r}(83)$ \\
\hline \multirow[t]{3}{*}{$495 W$} & & & & 484 & $\begin{array}{l}\mathrm{NC}^{a} \mathrm{C} d(30), \mathrm{CO} \mathrm{ib}(14), \mathrm{C}^{\delta} \mathrm{O}_{2} \mathrm{w}(12), \\
\quad \mathrm{C}^{a} \mathrm{C} s(11)\end{array}$ \\
\hline & $475 \mathrm{We}^{\mathrm{e}}$ & & & & $\delta\left(\mathrm{OC}^{8} \mathrm{O}\right)\left[478\left(\mathrm{~B}_{u}\right)\right]$ \\
\hline & $409 \mathrm{M}$ & & 402 & & $\begin{array}{l}\text { CO ib(15),NH ob(15),CNC } C^{\alpha} d(13) \\
C^{\alpha} \mathrm{CN} d(10), \mathrm{CO} \text { ob(10) }\end{array}$ \\
\hline \multirow[t]{4}{*}{$385 W$} & & & & 379 & $\begin{array}{l}\mathrm{C}^{\beta} \text { b1 }(20), \mathrm{C}^{\beta} \text { b2(13), } \mathrm{C}^{\alpha} \mathrm{C}^{\beta} \mathrm{C}^{\gamma} \mathrm{d}(13) \\
\mathrm{CNC}^{\alpha} \mathrm{d}(12)\end{array}$ \\
\hline & & 368 & & & $\begin{array}{l}\mathrm{CO} \text { ob }(17), \mathrm{C}^{\beta} \text { b1(14),NC } \mathrm{N}^{\alpha} \mathrm{C} d(11) \\
\mathrm{C}^{\beta} \mathrm{C}^{\gamma} \mathrm{C}^{\delta} \mathrm{d}(10), \mathrm{NH} \text { ob }(10)\end{array}$ \\
\hline & & & & 353 & $\mathrm{C}^{\beta} \mathrm{b} 2(31), \mathrm{C}^{\alpha} \mathrm{CN} \mathrm{d}(19)$ \\
\hline & & & 334 & & $\mathrm{C}^{\beta} \mathrm{b} 2(46), \mathrm{CO} \mathrm{ib}(11)$ \\
\hline $318 \mathrm{~W}$ & & 334 & & & $\begin{array}{l}\mathrm{CO} \mathrm{ib}(27), \mathrm{C}^{\beta} \text { b2(14), } \mathrm{CNC}^{a} \mathrm{~d}(10), \\
\quad \mathrm{C}^{\mathrm{a}} \mathrm{C}^{\beta} \mathrm{C}^{\gamma} \mathrm{d}(10)\end{array}$ \\
\hline \multirow[t]{4}{*}{$280 V W$} & & 265 & & & $\mathrm{C}^{\beta} \mathrm{b} 2(31), \mathrm{C}^{\beta} \mathrm{b} 1(13)$ \\
\hline & & & 240 & & $\begin{array}{l}\mathrm{C}^{\alpha} \mathrm{C}^{\beta} \mathrm{C}^{\gamma} \mathrm{d}(28), \mathrm{C}^{\beta} \mathrm{b} 1(18) \\
\mathrm{C}^{\beta} \mathrm{C}^{\gamma} \mathrm{C}^{\delta} \mathrm{d}(12)\end{array}$ \\
\hline & & & & 234 & $\mathrm{C}^{\alpha} \mathrm{C}^{\beta} \mathrm{C}^{\gamma} \mathrm{d}(38), \mathrm{C}^{\beta} \mathrm{b} 1(15), \mathrm{CO}$ ob(10) \\
\hline & & & & 219 & $\mathrm{C}^{\beta} \mathrm{C}^{\gamma} \mathrm{C}^{\delta} \mathrm{d}(22), \mathrm{C}^{\alpha} \mathrm{CN} \mathrm{d}(14), \mathrm{C}^{\beta} 2(10)$ \\
\hline \multirow{13}{*}{$204 \mathrm{VW}$} & & & 206 & & $\begin{array}{l}{ }^{\beta} \mathrm{C}^{\gamma} \mathrm{C}^{\delta} \mathrm{d}(15), \mathrm{C}^{\alpha} \mathrm{C}^{\beta} \mathrm{C}^{\gamma} \mathrm{d}(13) \\
\mathrm{C}^{\alpha} \mathrm{CN} \mathrm{d}(10)\end{array}$ \\
\hline & & 194 & & & $\mathrm{C}^{\alpha} \mathrm{C}^{\beta} \mathrm{C}^{\gamma} \mathrm{d}(38), \mathrm{C}^{\alpha} \mathrm{CN} \mathrm{d}(\mathbf{1 4})$ \\
\hline & & & 175 & & $\begin{array}{l}\mathrm{CNC}^{\alpha} \mathrm{d}(29), \mathrm{C}^{\alpha} \mathrm{CN} d(14) \\
\mathrm{C}^{\beta} \mathrm{C}^{\gamma} \mathrm{C}^{5} \mathrm{~d}(14), \mathrm{NH} \text { ob }(11)\end{array}$ \\
\hline & & & & 171 & $\begin{array}{l}\mathrm{NH} \text { ob(32), } \mathrm{C}^{a} \mathrm{CN} \mathrm{d}(18), \mathrm{CNC}^{\alpha} \mathrm{d}(15) \\
\quad \mathrm{C}^{\beta} \mathrm{C}^{\gamma} \mathrm{C}^{\delta} \mathrm{d}(13), \mathrm{C}^{\beta} \mathrm{b} 2(11)\end{array}$ \\
\hline & & 170 & & & $\mathrm{C}^{\beta} \mathrm{C}^{\gamma} \mathrm{C}^{\delta} \mathrm{d}(29), \mathrm{CNC}^{\mathrm{a}} \mathrm{d}(25)$ \\
\hline & & 130 & & & $\mathrm{C}^{\gamma} \mathrm{C}^{\delta} \mathrm{t}(41), \mathrm{C}^{\alpha} \mathrm{C}^{\beta} \mathrm{t}(12)$ \\
\hline & & & & 121 & $\mathrm{C}^{\gamma} \mathrm{C}^{\delta} \mathrm{t}(53), \mathrm{C}^{\alpha} \mathrm{C}^{\beta} \mathrm{t}(12)$ \\
\hline & & & 117 & & $\mathrm{C}^{\gamma} \mathrm{C}^{8}(40)$ \\
\hline & & & 115 & & $\mathrm{Cr}^{\gamma} \mathrm{C}^{\delta}(24)$ \\
\hline & & & & 109 & $\mathrm{C}^{\beta}$ b1 (19), CNC $\mathrm{C}^{a} \mathrm{~d}(11), \mathrm{C}^{\beta} \mathrm{C}^{\gamma} \mathrm{C}^{\delta} \mathrm{d}(10)$ \\
\hline & & 101 & & & $\mathrm{C}^{\gamma} \mathrm{C}^{6} \mathrm{t}(26), \mathrm{CN} \mathrm{t}(10), \mathrm{H} \cdots \mathrm{O} \mathrm{s}(10)$ \\
\hline & & & & 66 & $\begin{array}{l}\text { NH ob(20), } \mathrm{NC}^{\alpha} \mathrm{C} d(15), \mathrm{C}^{\beta} \mathrm{C}^{\gamma} \mathrm{t}(14), \\
\mathrm{H} \ldots \mathrm{O}(13)\end{array}$ \\
\hline & & & 64 & & $\begin{array}{l}\text { NH ob }(25), N C^{a} C d(14), C N t(14) \\
\quad C^{\beta} C^{\gamma} t(12)\end{array}$ \\
\hline
\end{tabular}


TABLE I (Continued)

Observed and Calculated Frequencies (in $\mathrm{cm}^{-1}$ ) of $\alpha$-Poly(L-Glutamic Acid)

\begin{tabular}{|c|c|c|c|c|c|}
\hline \multicolumn{2}{|c|}{ Observeda } & \multicolumn{4}{|r|}{ Calculated } \\
\hline $\operatorname{Raman}^{b}$ & ir & A & $\mathrm{E}_{1}$ & $\mathrm{E}_{2}$ & Potential Energy Distribution ${ }^{c}$ \\
\hline & & 60 & & & $\begin{array}{l}\mathrm{NC}^{a} \mathrm{t}(15), \mathrm{NC} \mathrm{C}^{\alpha} \mathrm{Cd}(14), \mathrm{C}^{a} \mathrm{C} t(14), \\
\mathrm{C}^{\alpha} \mathrm{C}^{\beta} \mathrm{C}^{\gamma} \mathrm{d}(12), \mathrm{CN} \mathrm{t}(12)\end{array}$ \\
\hline & & & & 39 & $\begin{array}{l}\text { NH ob }(37), C^{\beta} \text { b1 } 1(19), C^{\alpha} \mathrm{C} t(19) \\
\quad C N t(16), N^{\alpha} t(15), H \ldots O s(10)\end{array}$ \\
\hline & & 35 & & & $C^{\alpha} C^{\beta} t(45), C^{\beta} C^{\gamma} t(37), C^{\gamma} C^{\delta} t(11)$ \\
\hline & & & 35 & & $C^{\alpha} C^{\beta} t(51), C^{\beta} C^{\gamma} t(29), C^{\gamma} C^{\delta} t(12)$ \\
\hline & & & & 35 & $\mathrm{C}^{a} \mathrm{C}^{\beta} \mathrm{t}(46), \mathrm{C}^{\beta} \mathrm{C}^{\gamma} \mathrm{t}(31), \mathrm{C}^{\gamma} \mathrm{C}^{\delta} \mathrm{t}(12)$ \\
\hline & & & 23 & & $\mathrm{C}^{\beta} \mathrm{C}^{\gamma} \mathrm{t}(32), \mathrm{C}^{\alpha} \mathrm{C}^{\beta} \mathrm{t}(14)$ \\
\hline & & 21 & & & $\mathrm{C}^{\beta} \mathrm{C}^{\gamma} \mathrm{t}(42), \mathrm{C}^{\alpha} \mathrm{C}^{\beta} \mathrm{t}(28)$ \\
\hline & & & & 21 & $\begin{array}{l}\mathrm{C}^{\beta} \mathrm{C}^{\gamma} \mathrm{t}(28), \mathrm{NH} \text { ob(18), } \mathrm{C}^{a} \mathrm{C}^{\beta} \mathrm{t}(14), \\
\mathrm{NC}^{\alpha} \mathrm{C} d(10)\end{array}$ \\
\hline & & & 13 & & $\begin{array}{l}\text { NH ob(39), } C^{\beta} \text { b1(12), } C^{\beta} C^{\gamma} t(12), \\
C^{\alpha} C^{\beta} t(11), N^{\alpha} C d(11)\end{array}$ \\
\hline & & & & 11 & $\mathrm{C}^{\circ} \mathrm{C} \mathrm{t}(36), \mathrm{H} \ldots \mathrm{O} \mathrm{s}(18)$ \\
\hline
\end{tabular}

${ }^{\mathrm{a}} \mathrm{S}=$ strong, $\mathrm{M}=$ medium, $\mathrm{W}=$ weak, $\mathrm{V}=$ very, $\mathrm{sh}=$ shoulder.

b From Ref. 8.

${ }^{c} \mathrm{~s}=$ stretch, as = antisymmetric stretch, ss = symmetric stretch, $\mathrm{b}=$ angle bend, $\mathrm{ib}=$ in-plane angle bend, $\mathrm{ob}=$ out-of-plane angle bend, $\mathrm{w}=$ wag, $\mathrm{r}=$ rock, $\mathrm{tw}=$ twist, $\mathrm{d}=$ deformation, $\mathrm{t}=$ torsion. Only contributions of $10 \%$ or greater are included.

d Observed frequency, not corrected for Fermi resonance.

- Modes primarily due to vibrations of the $\mathrm{COOH}$ group. In the PED column, we indicate the coordinate having the maximum contribution and the observed frequency in the acetic acid dimer (Ref. 14).

'Dichroism data from Refs. 3-5.

8 From Refs. 3 and 7.

for anharmonicity effects, ${ }^{13}$ this frequency is still about $7 \mathrm{~cm}^{-1}$ lower than the value of $3279 \mathrm{~cm}^{-1}$ found for the unperturbed $\mathrm{NH}$ stretch mode in $\alpha$-(Ala $)_{n}{ }^{13}$ A possible explanation is that the hydrogen bond in $\alpha(\mathrm{GluH})_{n}$ is slightly stronger than that in $\alpha$-(Ala $)_{n}$.

The Raman and ir bands near $1720 \mathrm{~cm}^{-1}$ are clearly assignable to the hydrogen-bonded $\mathrm{C}=\mathrm{O}$ stretch in the $\mathrm{COOH}$ group: the comparable band in the acetic acid dimer is at $1739 \mathrm{~cm}^{-1} .{ }^{14}$ The slight parallel dichroism of this band ${ }^{3,5}$ indicates that the orientations of the $\mathrm{COOH}$ groups are such that (assuming uniaxial orientation) the $\mathrm{C}=\mathrm{O}$ bonds make an angle of slightly less than $54.7^{\circ}$ with the helix axis..$^{15}$ In view of the large dichroism of the amide I and amide II modes (see below), it is unlikely that the $\mathrm{C}=\mathrm{O}$ bonds are oriented as nearly parallel to the helix axis as is required by the model in which side-chain $\mathrm{COOH}$ groups form a hydrogen-bonded helix. ${ }^{16}$ In fact, in the solid state it is quite possible that interhelix $\mathrm{COOH}$ hydrogen bonds occur, perhaps in addition to intrahelix hydrogen bonds.

The A species amide I mode (primarily $\mathrm{C}=\mathrm{O}$ stretch) is observed as a highly parallel dichroic ${ }^{3-5}$ band at $1652 \mathrm{R}, 1653$ ir $\mathrm{cm}^{-1}$, compared 
with $1655 \mathrm{R}, 1658$ ir $\mathrm{cm}^{-1}$ in $\alpha$-(Ala) ${ }_{n} .{ }^{12}$ The $\mathrm{E}_{1}$ species amide II mode (primarily NH in-plane bend plus $\mathrm{CN}$ stretch) is found with high perpendicular dichroism ${ }^{3-5}$ at $1550 \mathrm{~cm}^{-1}$ in the ir, compared with 1545 $\mathrm{cm}^{-1}$ in $\alpha$-(Ala) ${ }_{n}{ }^{12}$ Both of these small shifts in frequency in $\alpha-(\mathrm{GluH})_{n}$ compared to $\alpha$-(Ala) ${ }_{n}$ are also consistent with a slightly stronger hydrogen bond in the former case and, taken together with the evidence from NH stretch, strongly support this possibility. The weak band observed at $1510 \mathrm{~cm}^{-1}$, and having parallel dichroism, 3,5 is reasonably accounted for by the A species amide II mode calculated at $1517 \mathrm{~cm}^{-1}$. (Note that predicted bands at $1561 \mathrm{~cm}^{-1}$ are a result of the assumption of a $\mathrm{COO}^{-}$group, which of course is not present. The $\mathrm{COOH}$ group that is present in $\alpha-(\mathrm{GluH})_{n}$ is responsible for the $1720 \mathrm{~cm}^{-1}$ band discussed above.)

The observed bands at $1450 \mathrm{R}, 1451 \mathrm{ir}$ and $1420 \mathrm{R}, 1417 \mathrm{ir} \mathrm{cm}^{-1}$ are well assigned to side-chain $\mathrm{CH}_{2}$ bend modes. In aqueous solution at low $\mathrm{pH}$, where $(\mathrm{GluH})_{n}$ is in an $\alpha$-helix conformation, the Raman bands shift down to 1440 and $1412 \mathrm{~cm}^{-1}{ }^{6}$ This may indicate that the sidechain conformation is different in the two cases, being not as fully extended in aqueous solution. We will examine other arguments pointing to this conclusion when we discuss the skeletal modes. (Note that predicted bands at $1416 \mathrm{~cm}^{-1}$ are a result of the assumption of a $\mathrm{COO}^{-}$ group. The Raman spectrum at high $\mathrm{pH}$, where the carboxyl groups are ionized, in fact shows a strong band at $1404 \mathrm{~cm}^{-1} .6$ )

The $\sim 1400-1200 \mathrm{~cm}^{-1}$ region is one in which side-chain modes significantly influence main-chain modes. This mixing is much greater than in the case of $\alpha$-(Ala) ${ }_{n}$, where, for example, the $\mathrm{CH}_{3}$ modes are completely separable from the amide III modes, ${ }^{12}$ a situation that is not true for $\alpha-(\mathrm{GluH})_{n}$. Nevertheless, the normal mode calculation gives good predictions of the observed bands. The weak ir band at 1388 $\mathrm{cm}^{-1}$ is reasonably assigned to $\mathrm{A}$ or $\mathrm{E}_{1}$ species modes (the observed dichroism ${ }^{3,5}$ is uncertain) that involve mixing of $\mathrm{C}^{\beta} \mathrm{H}_{2}$ wag with $\mathrm{H}^{\alpha}$ bend. The $1340 \mathrm{~cm}^{-1}$ Raman band (with a weak counterpart in the ir at $1343 \mathrm{~cm}^{-1}$ ) is observed to weaken slightly on $\mathrm{N}$-deuteration. ${ }^{8,11}$ The calculations predict that $\mathrm{E}_{1}$ and $\mathrm{E}_{2}$ modes at $1326 \mathrm{~cm}^{-1}$ contain $\mathrm{NH}$ in-plane bend contributions, while $\mathrm{C}^{\beta} \mathrm{H}_{2}$ twist plus $\mathrm{H}^{\alpha}$ bend modes are predicted at 1343 and $1341 \mathrm{~cm}^{-1}$. It is possible that the observed band at $1340 \mathrm{~cm}^{-1}$ contains an overlap of these two kinds of modes, thus weakening only partially on $\mathrm{N}$-deuteration. A similar explanation was suggested for the $1336 \mathrm{~cm}^{-1}$ band of $\alpha$-poly ( $\gamma$-benzyl-L-glutamate). ${ }^{17}$ The weak ir band at $1313 \mathrm{~cm}^{-1}$ appears to be unaffected by $\mathrm{N}$-deuteration and is well assigned to $\mathrm{C}^{\gamma} \mathrm{H}_{2}$ wag modes at $1307\left(\mathrm{E}_{1}\right)$ and $1305(\mathrm{~A}) \mathrm{cm}^{-1}$ (the observed dichroism is uncertain). On the other hand, the Raman band at $1296 \mathrm{~cm}^{-1}$ clearly weakens on $\mathrm{N}$-deuteration, ${ }^{8}$ as does our observed ir band at $1283 \mathrm{~cm}^{-1}$, and these are very well accounted for by the calculated modes at $1299\left(\mathrm{E}_{2}\right)$ and $1287\left(\mathrm{E}_{1}\right) \mathrm{cm}^{-1}$, respectively, that contain NH in-plane bend. (It is interesting to note that the com- 
parable bands in $\alpha$-(Ala $)_{n}$ are observed at $1278 \mathrm{R}$ and $1270 \mathrm{ir}^{-1}$ and are calculated at $1287\left(\mathrm{E}_{2}\right)$ and $1278\left(\mathrm{E}_{1}\right) \mathrm{cm}^{-1}$, respectively, ${ }^{12}$ clearly showing the influence of the side-chain structure on the main-chain amide III modes.) The $1253 \mathrm{~cm}^{-1}$ ir band shows no conspicuous change in N-deuteration; therefore, it is unlikely to be an amide III mode, as previously suggested. ${ }^{6}$ It is more likely to be due to $\mathrm{C}-\mathrm{O}$ stretch in the $\mathrm{COOH}$ group, which is found at $1255 \mathrm{~cm}^{-1}$ in the acetic acid dimer. ${ }^{14}$ The weak $1214 \mathrm{R}, 1212 \mathrm{ir} \mathrm{cm}-1$ bands are assignable to $\mathrm{CH}_{2}$ wag modes, despite the large discrepancy with the predicted frequencies near 1238 $\mathrm{cm}^{-1}$. This suggests that the $\sim 1238 \mathrm{~cm}^{-1}$ Raman band observed in $\alpha$ (GluH) ${ }_{n}$ at low $\mathrm{pH}^{6}$ should be assigned to $\mathrm{CH}_{2}$ wag and not amide III,

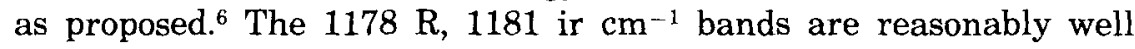
predicted and arise from $\mathrm{H}^{\alpha}$ bend plus $\mathrm{C}^{\beta} \mathrm{H}_{2}$ twist modes.

The $\sim 1100-900 \mathrm{~cm}^{-1}$ region is dominated by main-chain and sidechain backbone stretching vibrations. The bands in this region are not only well predicted by the calculation, but the differences, as compared with $\beta$-(GluCa $)_{n}$ and $\alpha-(\mathrm{Ala})_{n}$, are also accounted for. in $\alpha-(\mathrm{GluH})_{n}$, the $1117 \mathrm{R}, 1118 \mathrm{ir} \mathrm{cm}^{-1}$ bands are due to mixed $\mathrm{NC}^{\alpha}$ stretch and $\mathrm{C}^{\alpha} \mathrm{C}^{\beta}$ stretch modes calculated at $1129 \mathrm{~cm}^{-1}$. The same mixing occurs in $\beta$ (GluCa) ${ }_{n}$, but the $\mathrm{C}^{\alpha} \mathrm{C}^{\beta}$ stretch predominates ${ }^{1}$ and the mode is calculated at $1111 \mathrm{~cm}^{-1}$ but seems not to be observed. In $\alpha$-(Ala) $)_{n}, \mathrm{NC}^{\alpha}$ stretch mixes with $\mathrm{CH}_{3}$ rock and $\mathrm{C}^{\alpha} \mathrm{C}^{\gamma}$ stretch, ${ }^{12}$ with the result that the calculated and observed frequencies are near $1170 \mathrm{~cm}^{-1}$. The 1080 $\mathrm{R}, 1083 \mathrm{ir} \mathrm{cm}^{-1}$ bands of $\alpha-(\mathrm{GluH})_{n}$ are associated with a mainly $\mathrm{C}^{\alpha} \mathrm{C}^{\beta}$ stretch mode calculated at $1085(\mathrm{~A}) \mathrm{cm}^{-1}$. In $\beta$-(GluCa) ${ }_{n}$, this mode is significantly mixed with $\mathrm{C}^{\beta} \mathrm{C}^{\alpha}$ stretch, ${ }^{1}$ being calculated at $1062(\mathrm{~A})$ $\mathrm{cm}^{-1}$ and observed at $1066 \mathrm{~cm}^{-1}$. In $\alpha$-(Ala $)_{n}$, this mode is mixed with $\mathrm{CH}_{3}$ rock $^{12}$ and is calcualted at 1103 and observed near $1107 \mathrm{~cm}^{-1}$. Similarly, the $1057 \mathrm{R}, 1060 \mathrm{ir} \mathrm{cm}^{-1}$ bands of $\alpha-(\mathrm{GluH})_{n}$ are due mainly to $\mathrm{C}^{\beta} \mathrm{C}^{\gamma}$ stretch modes calculated near $1048 \mathrm{~cm}^{-1}$, which give rise to a medium-intensity Raman band. In $\beta$-(GluCa) ${ }_{n}$, this mode mixes with $\mathrm{C}^{\alpha} \mathrm{C}^{\beta}$ stretch and $\mathrm{C}^{\beta} \mathrm{H}_{2}$ rock, calculated at $1041 \mathrm{~cm}^{-1}$, and gives rise to a very weak Raman band at $1050 \mathrm{~cm}^{-1}$. The observed bands at 1002 $\mathrm{R}, 982$ ir $\mathrm{cm}^{-1}$ are associated with mixed $\mathrm{C}^{\gamma} \mathrm{C}^{\delta}$ and $\mathrm{C}^{\beta} \mathrm{C}^{\gamma}$ stretch, with calculated frequencies at $998\left(\mathrm{E}_{2}\right)$ and $996\left(\mathrm{E}_{1}\right), 992(\mathrm{~A}) \mathrm{cm}^{-1}$; in $\beta$ $(\mathrm{GluCa})_{n}$, the $\mathrm{C}^{\gamma} \mathrm{C}^{\delta}$ stretch is relatively unmixed ${ }^{1}$ and seems to be associated with calculated and observed frequencies at 1003 and $~ 1013$ $\mathrm{cm}^{-1}$, respectively. The calculations do not predict additional modes to which the weak Raman and ir bands near $1030 \mathrm{~cm}^{-1}$ could be assigned. The Raman spectrum of $\alpha$-(GluMg ${ }_{n}$ shows only a shoulder near this frequency whereas there is a pronounced band in $\alpha$ (GluCa) ${ }_{n} \cdot{ }^{11}$ It seems quite possible that such a band is associated with a side chain that is not fully extended. This band also is present in the Raman spectrum of $\alpha-(\mathrm{GluH})_{n}$ in aqueous solution at low $\mathrm{pH}^{6}{ }^{6}$ where we have seen that shifted $\mathrm{CH}_{2}$ bend frequencies also indicate a possible departure from a fully extended side chain. A strong Raman 
band in the $1000-900 \mathrm{~cm}^{-1}$ region is usually considered to be characteristic of the $\alpha$-helix structure. ${ }^{18}$ Such a band is found in $\alpha-(\mathrm{GluH})_{n}$ at $924 \mathrm{~cm}^{-1}$, in excellent agreement with the calculated mode at 922 $\mathrm{cm}^{-1}$. Note that this mode, with contributions in addition to $\mathrm{CN}$ stretch and $\mathrm{CNC}^{a}$ deformation, is predicted at $910 \mathrm{~cm}^{-1}$ in $\alpha$-(Ala $)_{n},{ }^{12}$ and is, indeed, found at a lower frequency, viz., $908 \mathrm{~cm}^{-1}$.

The 896 ir and $874 \mathrm{R}, 869 \mathrm{ir} \mathrm{cm}^{-1}$ bands can be correlated with $\mathrm{CC}$ stretch modes found in the acetic acid dimer. ${ }^{14}$ These, of course, correspond to our $\mathrm{C}^{\gamma} \mathrm{C}^{\delta}$ stretch, but the contribution of the latter may be modified by our assumption of a terminal $\mathrm{COO}^{-}$group. The $826 \mathrm{R}$, $824 \mathrm{ir}$ and the $810 \mathrm{ir} \mathrm{cm}-1$ bands can be assigned to predicted $\mathrm{CH}_{2}$ rock modes. Since the calculations do not predict a large splitting, it is possible that one of the above bands (probably near $825 \mathrm{~cm}^{-1}$ ) could be associated with modes of a disordered side chain. In $\alpha$-(Ala ${ }_{n}$, a medium intensity ir band at $774 \mathrm{~cm}^{-1}$ is assignable to an $\mathrm{E}_{1}$ species $\mathrm{C}=\mathrm{O}$ out-of-plane bend mode calculated at $780 \mathrm{~cm}^{-1}{ }^{12}$; we assign the 768 ir $\mathrm{cm}^{-1}$ band of $\alpha-(\mathrm{GluH})_{n}$ by analogy (although it may also be correlated with the $777 \mathrm{~cm}^{-1}$ A species mode). Similarly, in $\alpha$-(Ala) ${ }_{n}$

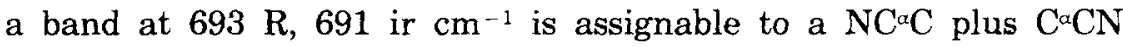
deformation mode calculated at ${ }^{12} 700(\mathrm{~A}) \mathrm{cm}^{-1}$; it seems reasonable to assign the $705 \mathrm{~cm}^{-1}$ band of $\alpha-(\mathrm{GluH})_{n}$ analogously (although there are some "fictitious" contributions of $\mathrm{C}^{\delta} \mathrm{O}_{2}$ bend and wag to the calculated $706 \mathrm{~cm}^{-1}$ mode).

The amide $\mathrm{V}$ modes (NH out-of-plane bend plus $\mathrm{CN}$ torsion) of $\alpha$ $(\mathrm{Ala})_{n}$ are calculated at $660\left(\mathrm{E}_{1}\right)$ and $608\left(\mathrm{E}_{1}\right) \mathrm{cm}^{-1}$, and they are observed as strong bands in the ir at 658 and $618 \mathrm{~cm}^{-1}$, respectively. ${ }^{12}$ In $\alpha$ $(\mathrm{GluH})_{n}$, these modes are calculated at 678 and $626 \mathrm{~cm}^{-1}$, and it seems reasonable to assign ir bands at 670 and $618 \mathrm{~cm}^{-1}$, respectively, to these modes. (It should be noted that the former band in not to be confused with a carbon dioxide band that occurs near this frequency, and whose small residual appearance has been subtracted out.) While the $618 \mathrm{~cm}^{-1}$ band clearly weakens on $\mathrm{N}$-deuteration, consistent with this assignment, it seems as if this is not the case for the $670 \mathrm{~cm}^{-1}$ band. This apparent discrepancy, in fact, demonstrates the importance of normal mode calculations in a complete analysis. We have computed the normal modes of the $\mathrm{N}$-deuterated molecule and find an interesting feature in the $700-600-\mathrm{cm}^{-1}$ region for the $\mathrm{E}_{1}$ species: a band is predicted at $662 \mathrm{~cm}^{-1}$, having a PED of $\mathrm{CO}$ ob(16) $\mathrm{NC}^{\alpha} \mathrm{C} \mathrm{d}(13) \mathrm{CO} \mathrm{ib}(13)$ $\mathrm{C}^{8} \mathrm{O}_{2} \mathrm{w}(12)$. (There is also a predicted mode at $613 \mathrm{~cm}^{-1}$ that is mainly $\mathrm{C}^{8} \mathrm{O}_{2}$ wag, as is the case for the $608 \mathrm{~cm}^{-1}$ mode of the protonated molecule.) This is somewhat analogous to the situation in $\alpha$-(AlaND) ${ }_{n}$, where a mode with a similar PED is predicted at $632\left(\mathrm{E}_{1}\right)$ and observed at $647{\mathrm{ir} \mathrm{cm}^{-1} .12}^{12}$ th the latter case, there is no overlap with either amide $\mathrm{V}$ mode of the protonated molecule, but for $\alpha-(\mathrm{GluH})_{n}$ such an overlap is indicated and probably accounts for the apparent lack of intensity decrease near $670 \mathrm{~cm}^{-1}$ on $\mathrm{N}$-deuteration. The amide $\mathrm{V}^{\prime}$ mode 
is predicted at $466\left(\mathrm{E}_{1}\right) \mathrm{cm}^{-1}$, and we suppose that the increased intensity in the $\mathrm{N}$-deuterated molecule at $475 \mathrm{~cm}^{-1}$ (where a bending vibration of the $\mathrm{COOH}$ group occurs) is due to the additional contribution from ND out-of-plane bend.

Special features of the $\alpha-(\mathrm{GluH})_{n}$ spectrum in the lower frequency region are also predicted reasonably well by the calculation. The 562 $\mathrm{R}, 567 \mathrm{ir} \mathrm{cm}^{-1}$ band is assignable to a highly mixed mode including an NH out-of-plane bend calculated at $549(\mathrm{~A}) \mathrm{cm}^{-1}$. A mode of similar character is calculated at $537(\mathrm{~A}) \mathrm{cm}^{-1}$ in $\alpha-(\mathrm{Ala})_{n}{ }^{12}$ and it has an observed counterpart at $530 \mathrm{R}, 526 \mathrm{ir} \mathrm{cm}-1$. The ir band at $409 \mathrm{~cm}^{-1}$ is uniquely assignable to the calculated $402\left(\mathrm{E}_{1}\right) \mathrm{cm}^{-1}$ mode of mixed character that also includes an NH out-of-plane bend. [A similar band is found in $\alpha$-helical polyglutamate esters, ${ }^{19}$ such as poly $(\gamma$-methyl-L-glutamate $\left(410 \mathrm{~cm}^{-1}\right)$, poly $\left(\gamma\right.$-benzyl-L-glutamate $\left(408 \mathrm{~cm}^{-1}\right)$, and poly $(\gamma-$ ethyl-L-glutamate) $\left(405 \mathrm{~cm}^{-1}\right)$.] A mode of almost identical character is calculated at $374\left(\mathrm{E}_{1}\right) \mathrm{cm}^{-1}$ in $\alpha$-(Ala $)_{n}{ }^{12}$ and it is observed at $375 \mathrm{R}$, ir $\mathrm{cm}^{-1}$. It is interesting that, although both modes contain a similar $\mathrm{C}^{\beta}$ bend 2 contribution, and other side-chain contributions in $\alpha-(\mathrm{GluH})_{n}$ are below the 10\% PED level $\left[\mathrm{C}^{\alpha} \mathrm{C}^{\beta} \mathrm{C}^{\gamma} \mathrm{d}(9) \mathrm{C}^{\beta} \mathrm{bl}(7) \mathrm{C}^{\beta} \mathrm{C}^{\gamma} \mathrm{C}^{\gamma} \mathrm{d}(6)\right]$, the frequencies are so different and this difference is so well accounted for. This testifies to the high reliability of the force field.

\section{CONCLUSIONS}

The normal modes of $\alpha-(\mathrm{GluH})_{n}$ have been calculated using the same helix structure and force field as for $\alpha-(\mathrm{Ala})_{n}{ }^{12}$ and a force field for an extended side chain transferred from $\beta$-(GluCa ${ }_{n} .{ }^{1}$ Despite an indication of a slightly stronger hydrogen bond in $\alpha-(\mathrm{GluH})_{n}$ than in $\alpha-(\mathrm{Ala})_{n}$, and therefore the possibility that a force field refined for the latter would not be completely satisfactory for predicting normal modes of the former, the observed Raman and ir bands are in general accounted for quite well. This indicates that the force field is highly reliable and transferable. In many cases, particularly in the amide III and 1100$900 \mathrm{~cm}^{-1}$ backbone stretching regions, significant differences are predicted and observed between the spectra of $\alpha-(\mathrm{GluH})_{n}$ and $\alpha-(\mathrm{Ala})_{n}$, thus clearly demonstrating the influence of the side chain on the vibrations of the main chain.

This research was supported by National Science Foundation Grants PCM-8214064 and DMR-8303610.

\section{References}

1. Sengupta, P. K. \& Krimm, S. (1984) Biopolymers 23, 1565-1594.

2. Krimm, S. (1983) Biopolymers 22, 217-225.

3. Ambrose, E. M. (1950) J. Chem. Soc. 3239-3249 (appendix to W. E. Hanby, S. G. Waley \& J. Watson).

4. Blout, E. R. \& Idelson, M. (1956) J. Am. Chem. Soc. 78, 497-498. 
5. Lenormant, H., Baudras, A. \& Blout, E. R. (1958) J. Am. Chem. Soc. 80, 61916195.

6. Koenig, J. L. \& Frushour, B. (1972) Biopolymers 11, 1871-1892.

7. Miyazawa, T. \& Blout, E. R. (1961) J. Am. Chem. Soc. 83, 712-719.

8. Fasman, G. D., Itoh, K., Liu, C. S. \& Lord, R. C. (1978) Biopolymers 17, 1729-1746.

9. Doty, P., Wada, A., Yang, J. T. \& Blout, E. R. (1957) J. Polym. Sci. 23, 851-861.

10. Zimmerman, S. S. \& Mandelkern, L. (1975) Biopolymers 14, 567-584.

11. Sengupta, P. K. \& Krimm, S. (1985) Spectrochim. Acta 41A, 205-207.

12. Dwivedi, A. M. \& Krimm, S. (1984) Biopolymers 23, 923-943.

13. Krimm, S. \& Dwivedi, A. M. (1982) J. Raman Spectrasc. 12, 133-137.

14. Nakamoto, K. \& Kishida, S. (1964) J. Chem. Phys. 41, 1558-1563.

15. Fraser, R. D. B. (1953) J. Chem. Phys. 21, 1511-1515.

16. Fasman, G. D. (1967) in Poly-a-Amino Acids, Fasman, G. D., Ed., Dekker, New York, p. 499.

17. Koenig, J. L. \& Sutton, P. L. (1971) Biopolymers 10, 89-106.

18. Frushour, B. G. \& Koenig, J. L. (1975) in Advances in Infrared and Raman Spectroscopy, Vol. 1, Heyden, London, pp. 35-97.

19. Miyazawa, T., Fukushima, K. \& Sugano, S. (1967) in Conformation of Biopolymers, Vol. 2, Ramachandran, G. N., Ed., Academic, New York, pp. 557-568.

Received January 2, 1985

Accepted April 19, 1985 